\title{
REPRESENTACIONES DE DOCENTES DE ESPAÑOL SOBRE LA CONSTRUCCIÓN DEL CONOCIMIENTO Y EL APRENDIZAJE EN EQUIPO
}

\section{REPRESENTATIONS OF SPANISH TEACHERS ABOUT KNOWLEDGE CONSTRUCTION AND LEARNING IN SMALL GROUPS OF STUDENTS}

Laura Rangel Bernal investigadora del departamento de Apoyo a la Formación Integral, Universidad Autónoma de Aguascalientes. laura.rangelb@gmail.com

\section{RESUMEN}

El objetivo de la investigación fue indagar las representaciones de docentes de español sobre la enseñanza y el aprendizaje de la lengua, para establecer cuáles propician procesos de construcción del conocimiento en alumnos de secundaria, al trabajar en equipos. Se realizó un estudio cualitativo mediante entrevista en profundidad y observación a dos docentes de la ciudad de Aguascalientes, México. Los resultados indican que los sujetos tienen conocimiento suficiente del modelo pedagógico, así como de los fundamentos epistemológicos en los que se basa éste; sin embargo, al aplicarlos en sus clases, ambos docentes presentan diferencias marcadas. Esto lleva a concluir que las formas en que los docentes intervienen para propiciar que se produzcan procesos 
constructivos en sus alumnos - cuando estos últimos realizan tareas de aprendizaje en equipo-, depende de cómo se representan los maestros tales constructos teóricos y de cómo los integran a su práctica.

Palabras clave: Pensamiento del profesor, construcción del conocimiento, enseñanza del español.

\section{ABSTRACT}

The aim of the investigation was to ascertain the representations of Spanish teachers about language teaching and learning in order to establish which of them promote processes of knowledge construction in their students as they work in small groups. A qualitative study through an in-depth interview and observations of two teachers from Aguascalientes, Mexico, was conducted. The results indicate that the teachers have sufficient knowledge about the pedagogic model of the subject they teach and about the epistemological principles in which such model is based upon. Nonetheless, when applied to their classes, both teaches differ considerably from one another. This leads to conclude that the ways in which these teachers intervene to promote processes of knowledge construction in their students when they engage in learning tasks while working in small groups, depends on the teachers' representations of these theoretical constructs and how they have integrated them to their teaching practice.

Key words: Pedagogical Content Knowledge, Constructivism (Learning), Spanish.

\section{INTRODUCCIÓN}

En 2006, con la reforma a la Educación Secundaria, se incorporaron el enfoque de las prácticas sociales del lenguaje y el método del trabajo por proyectos al modelo pedagógico de la materia de español. Con ello, la técnica didáctica (conocida como «trabajo en equipo») cobró una particular importancia, pues el mencionado modelo enfatiza la naturaleza social y constructiva, tanto de los objetos de conocimiento 
como de los procesos de enseñanza y aprendizaje de la lengua y, en consecuencia, promueve y prioriza el trabajo en conjunto sobre la modalidad individual.

Los conceptos teóricos y epistemológicos en los cuales se basa este modelo pedagógico desafían de diversas maneras a las formas más tradicionales de concebir la educación, formas que tienen un notable arraigo en México; debido a ello, transitar hacia ese modelo ha implicado retos importantes para todos los involucrados pero, de forma más significativa, para los docentes, quienes son los encargados de traducir estos constructos a términos prácticos y aplicarlos en sus clases.

Puesto que existe poca evidencia empírica sobre la forma en que se ha dado este tránsito - a pesar de haber transcurrido más de una década de su inicio-, y dado que el análisis de dicha evidencia es fundamental para evaluar la funcionalidad de cualquier modelo pedagógico, la investigación de la cual deriva este artículo tuvo como propósito indagar las representaciones de los maestros sobre un aspecto esencial para comprender el funcionamiento del modelo: la interrelación entre trabajo en equipo y construcción del conocimiento.

Esto, en el entendido de que dichas representaciones orientan la práctica docente de formas poco exploradas en investigaciones anteriores y de que, como lo indica Ferreiro (1998), es necesario estudiar los procesos educativos desde perspectivas que vayan más allá de los niveles pedagógico y didáctico ya que «cuando comprendamos a fondo las implicaciones epistemológicas de estos procesos habremos dado, quizás, un paso importante en la comprensión de la constitución de los objetos sociales en tanto objetos de conocimiento» (p. 154).

\section{EL TRABAJO EN EQUIPO COMO ESPACIO PARA LA CONSTRUCCIÓN DEL CONOCIMIENTO}

Para entender mejor la interrelación entre trabajo en equipo y construcción del conocimiento que aquí se aborda, es necesario conocer algunos planteamientos teóricos del modelo pedagógico de la materia de español. 
Desde 1993, los planes y programas de Educación Básica tienen como base un conjunto de postulados constructivistas que enfatizan el carácter social del aprendizaje. En lo que respecta a la materia de español, éstos establecen que: 1) «los alumnos son sujetos inteligentes susceptibles de adquirir, bajo procesos constructivos, el conocimiento y los usos de las diferentes manifestaciones sociales del lenguaje», y 2) «la lengua, oral y escrita, es un objeto de construcción y conocimiento eminentemente social, por lo que las situaciones de aprendizaje y uso más significativas ocurren en contextos de interacción social» (Secretaría de Educación Pública, 2011, p. 19).

Asimismo, en la reforma de 2006 se integró el enfoque de las prácticas sociales del lenguaje, concebidas como generadoras de situaciones de aprendizaje de la lengua que comprenden secuencias de actividades que integran procesos de producción lingüística y literaria, así como de reflexión metalingüística y metacomunicativa (Secretaría de Educación Pública, 2011).

Este enfoque resalta el carácter performativo del aprendizaje de la lengua, ya que se señala que éste se consigue únicamente a través de la participación activa de las personas en situaciones reales de interacción mediante el análisis, la interpretación, adaptación, transformación, producción y difusión de textos y los discursos de diversa índole (es decir, por medio del uso y movilización de los recursos lingüísticos que tienen a su disposición cuando interactúan en situaciones cercanas a su realidad cotidiana).

Trasladar estos planteamientos al salón de clases hace evidente que, para conseguir el aprendizaje de la lengua, se requiere de la interacción entre pares. En este sentido, el trabajo en equipo se vuelve un espacio privilegiado para que tanto los procesos constructivos como las prácticas sociales del lenguaje tengan lugar ya que, como lo señalan Ferreiro y Calderón (2000, p. 25), el trabajo en equipo o en pequeños grupos constituye, desde el punto de vista sociocognitivo, una forma de apropiación del conocimiento entre pares en tanto que permite a los alumnos reunirse para realizar diversas tareas de aprendizaje en torno al lenguaje y, en el proceso, negociar y asignar significados. 


\section{LAS REPRESENTACIONES DE LOS DOCENTES Y SU RELACIÓN CON LA PRÁCTICA}

Valorar las formas en que los docentes implementan un modelo pedagógico implica focalizar la mirada en acciones que, si bien son las manifestaciones concretas de la práctica, su análisis no alcanza para describir y explicar la génesis y el desarrollo de los problemas que surgen en dicho proceso. Para ello se requiere ver más allá de las acciones, indagar qué hay detrás de ellas, qué las motiva, qué las origina y de qué manera se puede intervenir con la finalidad de propiciar la reflexión sobre la práctica y, a partir de ello, promover su transformación.

Dado que buena parte de las respuestas a estas preguntas pueden hallarse en el pensamiento de los docentes, éste constituye un campo extenso de exploración e indagación. Debido a su naturaleza abstracta, se buscó un constructo teórico que sirviera para desagregar sus componentes a fin de conformar unidades discretas analizables y tratar de encontrar explicaciones a los problemas de enseñanza orientada a promover la construcción conjunta del conocimiento escolar. Un acercamiento a la teoría de las representaciones sociales proveyó dicho constructo.

El concepto de representaciones sociales fue propuesto por Serge Moscovici, en la década de 1960. Dicho autor, partiendo de principios ontológicos socioconstructivistas, concibe la realidad como resultado de un proceso histórico donde convergen diversos tipos de conocimiento (de forma más notable el científico y el popular), integrados de manera dialógica, dialéctica y dinámica. Con base en ello, Moscovici postula que, entre el sujeto y el objeto de conocimiento, media el cúmulo de conocimientos socialmente generados que posee una colectividad y que, de manera individual, posee cada persona. Este enfoque «reemplaza el dúo fundamentalista Ego-Objeto por la tríada Ego-Alter-Objeto» (Palmonari, en Romero, 2004, p. 256).

Se concibe entonces a las representaciones sociales como un punto de intersección entre el conocimiento científico y el conocimiento popular que permite a las personas simplificar teorías científicas complejas, así como conceptos demasiado abstractos o lejanos a la 
naturaleza propia de la constitución física humana y, de esta manera, integrar dichos conocimientos a su realidad cotidiana. Asimismo, las representaciones sociales constituyen un puente entre el conocimiento social y el individual, ya que establecen nexos de intersubjetividad, no solamente entre sujetos de un mismo grupo, sino entre distintos grupos humanos, tanto de forma sincrónica como diacrónica.

Debido a la diversidad de formas de conocimiento incluidas en las representaciones sociales, éstas constituyen un concepto integrador que contiene en sí mismo una forma de concebir, interpretar y conocer el mundo. Son, por tanto, un medio o una herramienta cognitiva a través del cual podemos comprender la realidad a partir del conocimiento compartido por una sociedad. Esto se refleja en las tres funciones epistémico-cognitivas que Jodelet asigna a las representaciones sociales: i) la función cognitiva, permite aprehender o conocer el mundo, partiendo de la base del conocimiento socialmente compartido; ii) la función interpretativa, a partir de la cual el individuo es capaz de asimilar dicho conocimiento, y generarse nociones y juicios de los objetos de conocimiento a través de la conformación de representaciones personales, y iii) la función de orientación de conductas y de relaciones sociales, mediante la cual las representaciones particulares modelan el comportamiento del individuo cuando interactúa con los objetos de conocimiento y con otros individuos (en Moscovici, 1988b).

La interrelación entre estas tres funciones explica por qué el conocimiento que posee un individuo no es exactamente el mismo que posee otro. Ocurre que cada sujeto, a partir de esta base común de conocimientos y de su experiencia vital, genera representaciones propias sobre el mundo, sobre sí mismo y sobre otros sujetos pues, de acuerdo con Jodelet (en Moscovici, 1988b), «incluso en representaciones muy elementales tiene lugar todo un proceso de elaboración cognitiva y simbólica que orientará comportamientos» (p. 478). Este planteamiento se conecta con el supuesto de que las personas no mantienen una postura neutra con respecto de los objetos que se representan, sino que sus representaciones generan actitudes que orientan patrones de conducta acordes con dichas actitudes. De este modo, representaciones diferentes generarán comportamientos diversos o, en el caso del problema que 
aquí se trata, prácticas docentes distintas. Es en este punto que se ubica la relación entre el contenido representacional y la práctica docente.

Como se aprecia, la teoría de las representaciones sociales proporciona un marco explicativo amplio para fenómenos de cognición, tanto individual como social, así como sobre prácticas de colectivos específicos entre los que se encuentra el profesorado. Por ello se decidió tomar al constructo de las representaciones sociales como referente principal para establecer la unidad de análisis de la investigación que, en este caso, delimitó estudiar a las representaciones de contenido epistemológico, es decir, aquellas que dan cuenta de las teorías o los supuestos teóricos a partir de los cuales los docentes explican cómo sus alumnos aprehenden los objetos de conocimiento. Tales representaciones sintetizan contenidos de carácter epistemológico, entrañados en el discurso de los docentes, y pueden identificarse en sus acciones orientadas a la enseñanza, así como en sus interacciones con el alumnado que dichos constructos orientan la práctica cotidiana de forma significativa, aunque no siempre sean explícitos.

En lo que respecta a la investigación educativa, autores como Mazzitelli, Aguilar, Guirado y Olivera (2009), Aguilar, Mazzitelli, Chacoma y Aparicio (2011), y Guirado, Mazzitelli y Olivera (2013) han estudiado la relación entre representaciones sociales y práctica docente en el ámbito de las ciencias naturales. Los resultados obtenidos por estas investigadoras dan cuenta de las representaciones que tienen los maestros sobre diversos aspectos de la educación, sobre el alumnado y sobre su papel como docentes y la relación que guardan dichas representaciones y la práctica docente. Sin embargo, la revisión de la literatura no condujo a ningún estudio que incorporara las categorías de trabajo en equipo y su relación con la construcción del conocimiento en el contexto específico de la enseñanza del español en secundaria, por lo que el presente estudio atiende a dicho vacío.

\section{METODOLOGÍA}

Dado que el objetivo de la investigación fue indagar las representaciones de docentes sobre la enseñanza y el aprendizaje del español 
para establecer cuáles propician procesos de construcción del conocimiento en alumnos de secundaria al trabajar en equipos, se optó por realizar un estudio cualitativo, considerando que éste permitiría analizar detalladamente tanto el discurso, como las acciones docentes.

La selección de los sujetos se llevó a cabo gracias a las facilidades otorgadas por las autoridades del Centro de Desarrollo Educativo «Jesús Terán», ubicado en el nororiente de la ciudad de Aguascalientes, México, y se realizó con base en los siguientes criterios: docentes en activo que impartieran la materia de español en cualquiera de los tres grados, en alguna de las secundarias adscritas a este centro; que aplicaran cotidianamente la técnica del trabajo en equipo en sus clases y que consintieran participar en el estudio.

De esta forma se visitaron siete escuelas públicas donde se contactaron a 30 docentes a quienes se les aplicó un cuestionario exploratorio que proveyó información sobre su formación, experiencia, lugar de trabajo, entre otros relacionados con su práctica docente cotidiana. De este grupo se eligieron dos sujetos con características similares: un maestro y una maestra, ambos egresados de la misma Escuela Normal quienes, al momento de la recopilación de los datos, contaban con estudios de posgrado en el área de educación y tenían menos de cinco años de haber empezado a laborar en el mismo plantel educativo.

Para la obtención de los datos se empleó la entrevista semiestructurada en profundidad y la observación abierta. Las entrevistas se realizaron en dos sesiones y comprendieron alrededor de 38 preguntas dirigidas a indagar las concepciones de los sujetos sobre el constructivismo, el trabajo en equipo y su conocimiento sobre los fundamentos pedagógicos y epistemológicos de la materia de español, de acuerdo con lo establecido en el plan de estudios y los programas de asignatura de la Secretaría de Educación Pública (2011).

Además se observaron 16 sesiones de clase a lo largo de dos meses. El registro de lo ocurrido fue sistemático, pues se contó con una lista de indicadores para orientar y focalizar la atención. Como soporte adicional, se videograbaron las sesiones de clase. Los videos resultantes se utilizaron para completar y precisar los registros escritos, además de que sirvieron para replantear y volver a analizar lo observado. 
La totalidad del material recabado fue transcrito antes de ser analizado, usando el método de la codificación abierta, propuesto por Strauss y Corbin (1998). El proceso fue el siguiente: se etiquetó y clasificó por fragmentos la información obtenida, se elaboró una tabla enlistando las categorías de investigación y en la que se consignaron los fragmentos transcritos correspondientes con cada categoría; se enunciaron las representaciones localizadas a través de un proceso de abstracción en el que se analizó lo que se dijo y observó, en relación con las categorías de investigación. Para esto se nombró cada una de las representaciones con una frase o una oración breve, de manera que pudieran identificarse y clasificarse dentro de las categorías iniciales.

En las tablas (una para cada docentes), se enunciaron las representaciones junto con la evidencia empírica que las sustenta y ejemplifica (fragmentos de las observaciones y de las entrevistas), agregando comentarios y explicaciones para clarificar su contenido. Una vez completadas las tablas de análisis de ambos sujetos, se procedió a redactar la descripción de cada una. La descripción se acompañó de argumentos teóricos y de elementos contextualizadores (explicaciones, precisiones, comentarios, etcétera), así como de fragmentos ilustrativos de las entrevistas y observaciones de clase realizadas.

Por último, cabe mencionar que el estudio realizado presentó algunas limitaciones como que, debido a que la participación de los docentes fue voluntaria y restringida a una sola zona escolar, pudo haberse producido un sesgo en la selección de éstos. Por otra parte, se abordó solamente el conocimiento conceptual, por tanto, a fin de obtener una visión más amplia sobre el tema, se recomienda confrontar los resultados con los de estudios que aporten datos sobre la construcción de otros tipos de conocimiento.

\section{RESULTADOS}

Los resultados de la investigación indican que los sujetos participantes cuentan con conocimiento suficiente del modelo pedagógico de la materia que imparten, así como de los fundamentos epistemológicos constructivistas en los cuales se basa dicho modelo. Sin embargo, en la 
puesta en práctica de estos constructos teóricos, ambos docentes presentan diferencias marcadas. La siguiente tabla incluye una síntesis de los resultados correspondientes a cada categoría, además de resaltar las diferencias encontradas entre los sujetos.

\section{Tabla. Representaciones de dos docentes de español sobre la construcción del conocimiento y el trabajo en equipo*.}

\begin{tabular}{|l|l|l|}
\hline $\begin{array}{l}\text { Representaciones } \\
\text { Construcción del } \\
\text { conocimiento }\end{array}$ & $\begin{array}{l}\text { Docente 1 } \\
\text { Construir es indagar y } \\
\text { reconstruir. }\end{array}$ & $\begin{array}{l}\text { Construir es inferir y } \\
\text { reproducir. }\end{array}$ \\
\hline $\begin{array}{l}\text { Conocimiento } \\
\text { escolar }\end{array}$ & $\begin{array}{l}\text { Es una reconstrucción de } \\
\text { contenidos socialmente } \\
\text { elaborados, por tanto, es } \\
\text { perfectible. }\end{array}$ & $\begin{array}{l}\text { Está preestablecido por } \\
\text { las autoridades en la } \\
\text { materia. Es concebido } \\
\text { como perfecto por tanto, } \\
\text { no puede ser construido, } \\
\text { sino reproducido. }\end{array}$ \\
\hline Aprendizaje & $\begin{array}{l}\text { Proceso de reconstrucción } \\
\text { del conocimiento escolar. }\end{array}$ & $\begin{array}{l}\text { Se constata mediante } \\
\text { la reproducción } \\
\text { de conceptos } \\
\text { preestablecidos. }\end{array}$ \\
\hline $\begin{array}{l}\text { Proceso de } \\
\text { aprendizaje }\end{array}$ & $\begin{array}{l}\text { Es evolutivo, dura toda } \\
\text { la vida y es de carácter } \\
\text { reconstructivo. Para } \\
\text { avanzar se requiere } \\
\text { reconstruir el conocimiento } \\
\text { previo y adquirir } \\
\text { habilidades metacognitivas } \\
\text { para aprender a aprender. }\end{array}$ & $\begin{array}{l}\text { Es inferencial y a } \\
\text { través de él se arriba al } \\
\text { conocimiento. }\end{array}$ \\
\hline
\end{tabular}




\begin{tabular}{|l|l|l|}
\hline Enseñanza & $\begin{array}{l}\text { Forma de mediar el proceso } \\
\text { de cambio conceptual que } \\
\text { Ilevan a cabo los alumnos } \\
\text { en interacción con sus } \\
\text { pares. }\end{array}$ & $\begin{array}{l}\text { Trazo de una ruta que los } \\
\text { alumnos deben seguir, } \\
\text { de forma individual, para } \\
\text { lograr el aprendizaje. }\end{array}$ \\
\hline $\begin{array}{l}\text { Metodología de la } \\
\text { enseñanza }\end{array}$ & $\begin{array}{l}\text { Es investigativa y pretende } \\
\text { promover la reconstrucción } \\
\text { de elaboraciones } \\
\text { conceptuales individuales. }\end{array}$ & $\begin{array}{l}\text { Es inferencial y pretende } \\
\text { conseguir que los } \\
\text { alumnos sean capaces de } \\
\text { reproducir conceptos. }\end{array}$ \\
\hline $\begin{array}{l}\text { Papel de los } \\
\text { docentes en la } \\
\text { enseñanza }\end{array}$ & $\begin{array}{l}\text { El docente media los } \\
\text { procesos cognitivos de los } \\
\text { alumnos que conducen } \\
\text { a la construcción del } \\
\text { conocimiento. }\end{array}$ & $\begin{array}{l}\text { El docente guía los } \\
\text { procesos educativos. }\end{array}$ \\
\hline $\begin{array}{l}\text { Papel del } \\
\text { alumnado en } \\
\text { el proceso de } \\
\text { aprendizaje }\end{array}$ & $\begin{array}{l}\text { Activo: se considera a los } \\
\text { alumnos como punto de } \\
\text { partida del proceso; se les } \\
\text { trata y caracteriza como } \\
\text { sujetos agentes, capaces } \\
\text { de regular sus propios } \\
\text { procesos de aprendizaje. }\end{array}$ & $\begin{array}{l}\text { Pasivo: se les trata y } \\
\text { caracteriza como sujetos } \\
\text { pacientes, aún sin la } \\
\text { capacidad de regular } \\
\text { sus propios procesos de } \\
\text { aprendizaje. }\end{array}$ \\
\hline $\begin{array}{l}\text { Perspectivas } \\
\text { didáctica y } \\
\text { pedagógica }\end{array}$ & $\begin{array}{l}\text { Centradas en el alumno y } \\
\text { en el aprendizaje. }\end{array}$ & $\begin{array}{l}\text { Centradas en el docente y } \\
\text { en la enseñanza. }\end{array}$ \\
\hline $\begin{array}{l}\text { El trabajo en } \\
\text { equipo y su papel } \\
\text { de la construcción } \\
\text { del conocimiento } \\
\text { en situaciones de } \\
\text { interacción entre } \\
\text { pares }\end{array}$ & $\begin{array}{l}\text { El trabajo en equipo } \\
\text { favorece los procesos } \\
\text { cognitivos individuales } \\
\text { co los alumnos, ya que } \\
\text { y promueve el aprendizaje } \\
\text { autónomo. }\end{array}$ & $\begin{array}{l}\text { El trabajo en equipo } \\
\text { cumple funciones } \\
\text { operativas. Se le } \\
\text { incorpora a las } \\
\text { estrategias didácticas } \\
\text { en la medida en que a la } \\
\text { docente le facilita tareas } \\
\text { relacionadas con la } \\
\text { enseñanza. }\end{array}$ \\
\hline
\end{tabular}

*Elaboración propia. 
Adicionalmente, se encontró que al aplicar la técnica del trabajo en equipo en sus clases, la intervención de los docentes no siempre aporta los elementos que propicien procesos de construcción del conocimiento en sus alumnos. Para ampliar e ilustrar estos puntos, se incluye a continuación un análisis de las categorías las cuales, para efectos de su presentación en este texto, se agruparon tomando en cuenta contenidos temáticos similares.

\section{¿Qué significa construir?}

En la entrevista, se pidió a los docentes explicar qué entienden por construcción del conocimiento. Sus respuestas integran diferentes elementos que es pertinente analizar. Por ejemplo, el docente 1 afirma que:

[...] construir le permite al sujeto, eh... hacer gala, utilizar todos aquellos recursos que tiene para poder aprender a aprender. Es decir, que sepa qué sabe y además de eso que sabe, sepa cómo aprender más... relacionado a que el sujeto construya su propio aprendizaje (entrevista, docente 1).

Resaltan tres aspectos en este fragmento. Primeramente, el maestro relaciona la capacidad de construir conocimiento con la competencia para lo que el programa de materia denomina aprendizaje permanente (Secretaría de Educación Pública, 2011: 39), en dos niveles: el metacognitivo («que sepa qué sabe») y la autogestión del conocimiento («y además de eso que sabe, sepa cómo aprender más»). Dichos elementos se conectan con la noción de agencia del sujeto cognoscente («que el sujeto construya su propio aprendizaje»), que es una de las bases del enfoque constructivista.

La mención de estos elementos es relevante ya que informa sobre los mecanismos a través de los cuales se pretende lograr una de las principales metas de la educación básica: que los estudiantes sean capaces de aprender de manera autónoma (Secretaría de Educación Pública, 2011). Asimismo, al mencionarlos, es de notarse que 
el docente 1 ubica al sujeto cognoscente (el alumno) en el centro de los procesos educativos, pues lo considera punto de partida y generador de los mismos. Lo anterior coincide con los planteamientos constructivistas del modelo educativo actual que considera al estudiante como «el centro y el referente fundamental del aprendizaje» (Secretaría de Educación Pública, 2011: 26).

Por su parte, la docente 2 define la construcción de conocimiento en los siguientes términos:

Construir implica formular a través de pequeñas... Es decir, a los alumnos... plantearles pequeños retos a través de pistas, a través de material que les obligue a inferir. Ya no es, en ese sentido, ya no se les da la información como antes, y se anota y se explica, sino que buscas el modo de que ellos tengan la posibilidad de ir armando, a través de estas pequeñas reflexiones e inferencias, un conocimiento que no tengas que dárselos tal como es, o como esperas que ellos lo tengan. [...] Así entiendo el constructivismo: el darles herramientas que les permitan a ellos inferir y llegar a ciertas conclusiones o al contenido que nosotros esperamos que ellos encuentren (entrevista, docente 2).

En su explicación, esta profesora se refiere a aspectos didácticos: cómo presentar la información y cómo conseguir que los alumnos la aprendan. Asimismo, esboza su papel como docente mencionando que su función es brindar herramientas para que los alumnos lleguen, a partir de inferencias, a conseguir los aprendizajes esperados.

De esta manera, se deja ver que el concepto de construcción que elabora la maestra está centrado en el docente y enfocado en la enseñanza, además de que se aleja de la concepción constructivista que manifiesta el docente 1 y se acerca más a una de tipo asociacionista o cognitivista, la cual considera necesaria la utilización de pistas («cues») a partir de las cuales los alumnos puedan realizar inferencias y, de este modo, conecten información nueva con información preexistente (Ertmer y Newby, 2013). 


\section{¿Qué es el conocimiento escolar y cómo se aprende?}

El docente 1 caracteriza al conocimiento escolar (entendido como aquel que se aprende en la escuela a partir de un currículum oficial determinado) como perfectible, inacabado, objeto en construcción y reconstrucción, susceptible de ser ampliado, precisado y reestructurado (entrevista, docente 1).

Una manifestación de esto, observada en su práctica, se encuentra en el hecho de que el maestro evita señalar errores o incorrecciones tanto en las aportaciones orales, como en los escritos elaborados por sus alumnos y, si los localiza, evita calificarlos como fallas o desaciertos, pues los reconoce como deficiencias que pueden subsanarse por los mismos alumnos y que se deben a que el aprendizaje aún está en proceso.

Este profesor conecta su manera de concebir el conocimiento escolar con elementos de la pedagogía y la didáctica del español. Esto lo hace, en el fragmento siguiente, comparando su propia concepción con una más tradicional en lo que respecta a la enseñanza y el aprendizaje de la lengua, por medio de la redacción de textos:

[...] hace algunos años, todavía era fundamental que un niño pudiera entregar un texto o, incluso un borrador de texto, sin faltas de ortografía. Si las tenía, tú se las corregías ahí mismo. Entonces, el nuevo enfoque ahora dice: bueno, sí es importante una corrección de ese tipo, pero no es lo más importante. O sea, tendrá su momento para hacer su corrección, hará una búsqueda en el diccionario para localizar palabras donde tenga duda, revisará con sus compañeros si... tiene alguna palabra escrita mal, su compañero le podrá corregir. Ya no todo girará alrededor de lo que es el docente, sino que ya hay una responsabilidad del aprendizaje en los alumnos (entrevista, docente 1).

Declaraciones como ésta, aunadas a lo observado en las clases, permiten afirmar que el maestro reconoce de forma consistente la facultad de agencia de sus alumnos y, por ende, su capacidad para realizar acciones que conduzcan a la construcción del conocimiento escolar. Esto 
concuerda con su aseveración de que los alumnos están en el centro de los procesos escolares y, en tanto, son responsables de su propio aprendizaje.

En el caso de la docente 2, su concepción de conocimiento escolar es distinta pues ella, como se mencionó en el apartado anterior, caracteriza los procesos constructivos como inferenciales. Esta concepción puede ilustrarse con una alegoría en la que se representa al aprendizaje como el recorrido de un camino que nos conducirá a un punto esperado - en este caso, al conocimiento escolar- y a la enseñanza como la labor de dejar pistas o indicios a lo largo de dicho camino para facilitar el trayecto. En consecuencia, la planeación didáctica es entendida como el trazo de la ruta a seguir. Dado que estas acciones están a cargo del docente, el rol del alumno es pasivo aunque se le dé un pequeño margen de acción.

Adicionalmente, tras esta concepción subyace una representación del conocimiento escolar como unívoco: el conocimiento escolar es el proporcionado por la docente quien, a su vez, se basa en fuentes autorizadas para delimitarlo y llevarlo a la clase, de modo que los alumnos no tienen margen para construirlo (en el sentido de asignarle significado): arribarán a él por medio de inferencias y su aprendizaje se corrobora si son capaces de reproducirlo.

Se observaron indicios de esta representación en sus clases, particularmente en una de ellas en que la maestra implementó una actividad consistente en que los alumnos formaran pares, juntando tarjetas que tenían anotado un término relacionado con el tema de la clase, con otras que contenían las definiciones de dichos términos. El fragmento que sigue ilustra cómo realizaron esta actividad un grupo de alumnos.

[La maestra se acerca al grupo de alumnos y se pone en cuclillas frente a las dos hileras de tarjetas que estos últimos han acomodado en el suelo] Docente 2: ¿Ya?, ¿ya creen tenerlo bien? Okey... Bien, me van a quitar la tarjeta de prólogo [Un alumno quita la tarjeta que tiene escrita la palabra prólogo]. La tarjeta no, mijo, la definición [este alumno mueve entonces la tarjeta que tiene escrita la definición]. Sí, gracias, y la de... 
[No se entiende en la grabación lo que dice la maestra, pero el alumno mueve otra tarjeta que tiene anotada una definición. La maestra revisa de nuevo la corrección de los pares que quedaron en el suelo y sonríe, aparentemente complacida. Los alumnos frente a ella, al ver su expresión, también sonríen].

Docente 2: Tienen dos mal, se quedarían con los doscientos puntos. ¿Qué se hace con esas dos? [Señala las que están incorrectas] Ya vieron cuáles eran. [El alumno que retiró y tiene en la mano las tarjetas incorrectas, las intercambia y ahora tienen todos los pares correctos] (Observaciones, docente 2).

Es importante señalar que los alumnos obtuvieron los puntos de calificación asignados por su habilidad de hacer coincidir las tarjetas con su definición mediante inferencias que no implicaron un proceso de construcción. El procedimiento es el siguiente: la maestra proporciona algunas pistas (palabras clave o «cues») que conectan las definiciones con los nombres de los términos y los alumnos siguen dichas pistas para encontrar la correspondencia entre las tarjetas. El momento más evidente de ausencia de indicios de construcción es al final del fragmento, donde, sin siquiera releer o analizar las tarjetas, con un simple cambio de orden, los pares quedan intercambiados y correctos. Posteriormente, los alumnos se limitaron a anotar esas definiciones en su cuaderno.

Se observó el uso de una estrategia didáctica que la maestra califica como más «dinámica» respecto de técnicas tradicionales, como la exposición o el dictado. Sin embargo, esta actividad no implica un cambio epistemológico de fondo. Su concepción de aprendizaje es que los alumnos tienen que arribar, de una forma más dinámica si se quiere, pero igualmente predeterminada, a contenidos conceptuales establecidos por ella quien a su vez, recurre a definiciones también preestablecidas, por lo que no tiene lugar entre los alumnos la indagación, negociación y asignación de significados, que son fundamentales en los procesos de aprendizaje entendidos en términos constructivistas. 


\section{La metodología de la enseñanza y el papel de los docentes}

En lo que respecta a la metodología de la enseñanza, el docente 1 tiende a priorizar la realización de actividades como la investigación ya que, como él lo afirma, el investigar permite a los alumnos buscar información en diversas fuentes, organizarla, clasificarla, evaluarla, etcétera, lo cual contribuye al aprendizaje en tanto que, al confrontar información surgida de diferentes fuentes, los alumnos realizan procesos de reconstrucción conceptual mediante los cuales pueden precisar y ampliar su conocimiento inicial sobre un tema dado (entrevista, docente 1).

De este modo, el maestro relaciona la actividad de investigar con el trabajo autónomo que, según declara, se requiere para promover la construcción del conocimiento y la autogestión del aprendizaje. En este sentido, sus intervenciones suelen estar dirigidas a promover que los alumnos se involucren en tareas de investigación cuando trabajan en equipos, mientras que su función como docente es fundamentalmente la de mediador:

[...] actualmente te puedo decir, de acuerdo con mi experiencia, que... es mucho más clara mi función bajo ese paradigma del aprendizaje: es que yo pueda mediar esos procesos, es decir, reconocer qué sabe el niño desde su primer acercamiento al contenido, a la materia, al objeto de aprendizaje, y a partir de eso, ir generando procesos que le permitan a él ir construyendo constantemente, de manera consciente, su propio aprendizaje, que es otro gran reto (entrevista, docente 1 ).

Sin embargo, es importante señalar que, de acuerdo con el modelo pedagógico, no todos los aprendizajes esperados se logran mediante una investigación, sino que para ello se requiere realizar diferentes tareas que involucren procesos cognitivos de diversa índole.

Lo que se observó es que el maestro prioriza la parte investigativa de los proyectos didácticos, ya que él la considera el eje de estos últimos. Sin embargo, al realizar los proyectos, se produce una desarticulación entre contenidos, procesos y productos lo cual deja de lado el 
trabajo en el área de las competencias lingüísticas, particularmente en lo respectivo a la lectoescritura y la comunicación oral, puesto que no se atienden estos aspectos de forma puntual, sino que se abordan como secundarios a las tareas de investigación.

Aunado a esto, el hecho de que el profesor evita sistemáticamente señalar errores o fallas, así como precisar términos a fin de no imponer su palabra sobre la de los alumnos, tiene como consecuencia que éstos no cuenten con parámetros o indicadores claros a partir de los cuales evaluar y gestionar sus aprendizajes del modo en que el profesor lo plantea.

En el caso de la docente 2, esto difiere considerablemente pues ella asume las principales funciones en lo que respecta a los procesos educativos. Por ejemplo, en una de sus clases, la maestra pidió a los alumnos reunirse en equipos para realizar una actividad que consistió en la lectura y el análisis de un cuento.

Después de repartir el material, ella leyó el cuento completo en voz alta mientras los alumnos seguían la lectura en silencio. Posteriormente, por medio de algunas preguntas dirigidas al grupo - pero que sólo unos pocos contestaron-, ella procedió a analizar el cuento según lo establecido en el programa de la materia, en tanto que los alumnos se limitaron a copiar en sus cuadernos lo que ella escribía en el pizarrón, sin que hubiera más aportaciones de parte de los primeros.

De este modo, se observó que la maestra se coloca a sí misma en el centro de los procesos educativos y asume las principales funciones en los mismos y, debido a ello, no existe margen para que los alumnos construyan mediante la asignación de significados, ya que no se promueve la realización de tareas que propicien tales procesos.

\section{El trabajo en equipo y la construcción del conocimiento}

El docente 1 manifiesta una opinión favorable respecto del trabajo en equipo. Esto se hace evidente en sus respuestas a la entrevista donde resalta los beneficios y las ventajas que dicha técnica le brinda. 
Al respecto señala que, desde que comenzó a trabajar en educación básica, ha considerado al trabajo en equipo como un elemento vital en la enseñanza del español, pues permite a los alumnos socializar más allá de la convivencia cotidiana con algunos compañeros, además de permitirles darse cuenta de sus propios procesos de aprendizaje y compararlos con los de otros, propiciando procesos metacognitivos y de reconstrucción y reestructuración del conocimiento. Por esta razón, el profesor considera que el trabajo en equipo empareja el avance de los alumnos, evitando que algunos se rezaguen (entrevista, docente 1 ).

Además, el maestro señala que el trabajo en equipo le permite observar los procesos de los alumnos con mayor facilidad que el trabajo grupal:

[...] ahí vienen otros beneficios que desde mi perspectiva encontramos o encuentro, y es el hecho de poder manejar más claro la organización y poder ver más preciso los procesos de los propios muchachos. Es decir, cuando yo me paro y hago una clase expositiva, yo la verdad no alcanzo a percibir si los cuarenta muchachos o, me atrevo a decir que ni el cincuenta por ciento de ellos, puedo yo alcanzar a visualizar o a identificar si los muchachos están en ese proceso y no me da posibilidades de manera particular o de manera individual de poder atender su propia necesidad (entrevista, docente 1).

Pese a que el maestro afirma que, al trabajar en equipo y seccionar al grupo, es más fácil percibir los procesos individuales y atender las necesidades particulares de los alumnos, en las observaciones realizadas no se tiene registro de que el docente haya abordado alguno de estos procesos de manera directa con algún alumno que lo requiriera.

Adicionalmente no se registraron, en los instrumentos de evaluación provistos por el profesor, elementos que indicaran que él habría de evaluar los procesos individuales que tuvieron lugar mientras se trabajaba en equipos de modo que, ni en su intervención ni en la evaluación, se encontraron elementos que sustentaran su afirmación.

En este punto es importante señalar que el docente 1 sí reconoce que el empleo de esta técnica didáctica no asegura conseguir los resultados 
de aprendizaje esperados al final de los proyectos. Aun así, no le atribuye los resultados insuficientes a las condiciones de aprendizaje que genera el trabajo en equipo, sino más bien a las deficiencias producidas en los procesos de interacción que pudieron darse entre los alumnos:

\begin{abstract}
Vamos, siento yo... creo que no garantiza que un equipo se reúna y entregue... no garantiza un producto o lo que se entregue, o una construcción cognitiva adecuada, sino que ésta se va dando conforme los niños van teniendo esa serie de experiencias de forma paulatina en ese proceso. Entonces, sí reconceptualizan, pero muchas veces no aterrizamos o no se aterriza. Sí producen, pero muchas veces lo que se produce... está todavía como un material en proceso, que todavía tiene características para poder mejorar (entrevista, docente 1).
\end{abstract}

De esta manera, el profesor establece que el potencial y la funcionalidad del trabajo en equipo no residen totalmente en la técnica misma, sino en quiénes integran el equipo y cómo interactúan entre sí, aunado a las formas y los grados de intervención docente.

La docente 2, por su parte, posee una percepción negativa sobre el trabajo en equipo, ya que considera que éste genera distracción, desorden, indisciplina, pérdida de tiempo, poca eficiencia y resultados de aprendizaje inferiores a los del trabajo individual lo que, a su vez, genera en ella emociones como frustración, incertidumbre e inclusive, vulnerabilidad:

Sí, yo antes pensaba realmente... me parecía más organizado el trabajo de manera individual, de forma que yo tuviera todo el control y todo el dominio de... tanto del contenido, como de la clase. Me parece que el cambio particular que hubo, y el miedo que, yo de manera particular me enfrento, es a que se te presentan muchísimas circunstancias para las que muchas veces no te encuentras preparado, circunstancias que en la anterior [reforma] no era tanto, porque el muchacho no entraba tanto en juego: era más pasiva su conducta en la clase y se sujetaba a los lineamientos y a las cuestiones conceptuales que tú le manejabas. Entonces te enfrenta ahora no sólo a que está construyendo su propio conocimiento, sino que también te plantea desafíos dentro de las clases. Entonces él te 
va a preguntar $y$, en muchas ocasiones, incluso te puede llegar a hacer preguntas para las que... para las que no estás, a la mejor, tan preparado (entrevista, docente 2).

En la representación de la maestra permea una concepción tradicional de la educación según la cual se espera que los alumnos trabajen de manera individual y en silencio, bajo la estricta guía de los profesores, mientras que los intercambios que se susciten entre ellos son considerados alteraciones al orden sancionables y que deben ser evitadas en la medida de lo posible. Esta concepción suele ir acompañada de juicios de valor respecto del desempeño docente: quien logra mantener el orden en su clase es un buen maestro, mientras que aquél al que se le dificulta o descuida este aspecto, es deficiente o malo ${ }^{1}$. Este tipo de representaciones limitan de forma considerable la interacción entre pares dentro del aula en favor de una concepción tradicional de disciplina escolar.

Por otra parte, la maestra dice reconocer los beneficios y otras características ventajosas que tiene la implementación del trabajo en equipo en sus clases. Por ejemplo, señala que el trabajo en equipo reduce la cantidad de trabajos por revisar, que facilita la distribución de tareas y materiales, que agiliza los procesos de realización de tareas y disminuye el número de dudas que puedan surgir en el grupo y que ella deba resolver (entrevista, docente 2).

Es importante resaltar que las ventajas que la maestra aduce están directamente relacionadas con la enseñanza y con la facilitación de sus tareas como docente, no con los alumnos ni lo correspondiente a sus procesos de aprendizaje. En este sentido, se observó que la maestra emplea el trabajo en equipo en sus clases en función de lo que esta técnica le permite o facilita a ella, de modo que, el potencial que tiene el trabajo en equipo como espacio para la construcción del conocimiento escolar entre pares, se desvanece.

1 Esto se relaciona con el concepto de gramática de la escolaridad que, de acuerdo con Tyack y Cuban (2000), constituye todos aquellos elementos que desde el siglo XIX se asocian con el deber ser de la escuela; es decir, lo que se cree que es una buena escuela, una representación que sigue muy arraigada en esta sociedad. 


\section{CONSIDERACIONES FINALES}

A modo de recapitulación, se retoman los hallazgos más relevantes:

En el caso del docente 1 destaca el hecho de que: i) éste concibe a los alumnos como el centro de los procesos escolares; ii) reconoce de forma consistente la capacidad de los mismos para construir el conocimiento escolar mediante procesos metacognitivos, de negociación y asignación de significados, y de autogestión del aprendizaje y, iii) entiende al trabajo en equipo como un espacio para el aprendizaje entre pares y lo prefiere sobre la modalidad individual.

Estos puntos coinciden con lo que plantea el modelo educativo y también concuerda con que, al implementar el trabajo en equipo, se brindaron más oportunidades para el surgimiento de procesos constructivos entre sus alumnos (en comparación con la docente 2), oportunidades que, sin embargo, se vieron limitadas puesto que este maestro no establece parámetros claros para que los alumnos evalúen sus propios aprendizajes, además de que, puesto que los proyectos didácticos dependen casi en su totalidad de la realización de tareas de investigación, se dejan de lado otros aspectos relativos al desarrollo de la lectoescritura y la comunicación oral.

Lo anterior contrasta con el caso de la docente 2, la cual: i) concibe los procesos constructivos como inferenciales; ii) se enfoca consistentemente en la enseñanza, colocándose a ella misma en el centro de los procesos educativos $y$, iii) reconoce el valor del trabajo en equipo en función de lo que éste le facilita en términos de su labor docente, al tiempo que prefiere la modalidad individual por considerar que sus resultados son superiores a los del trabajo en equipo. Por ende, las oportunidades para que los alumnos pudieran construir el conocimiento escolar al trabajar en equipo en sus clases fueron muy limitadas, dado que no se propiciaron las condiciones necesarias.

El análisis de estos resultados permite afirmar que existe una relación entre las representaciones que tienen los sujetos sobre los constructos que conforman la base teórica y epistemológica del modelo pedagógico de la materia de español, y las formas de intervención docente que propician la aparición de procesos de construcción del conocimiento en alumnos de secundaria, cuando éstos últimos trabajan en equipo. 
Esta relación parece explicar no sólo las acciones de cada sujeto, sino también las diferencias que se encontraron entre ellos, las cuales son notables considerando que ambos tienen características muy similares en cuanto a su formación, experiencia profesional y lugar de trabajo.

Estos puntos conducen a una reflexión sobre la manera en la que se implementan los nuevos modelos pedagógicos en México y a concluir que su mera institucionalización no redunda en una implementación exitosa, pues para ello se requiere, entre otras cosas, una reconfiguración completa del pensamiento docente que conlleve no sólo adecuaciones conceptuales sino, fundamentalmente, un cambio de tipo epistemológico.

Por ello, es importante enfatizar la necesidad de realizar más investigaciones que abonen al entendimiento de la compleja relación existente entre pensamiento y práctica docente, investigaciones que, a su vez, sirvan como base para el diseño de programas de formación e intervención que permitan a los maestros trabajar sobre sus propias representaciones, pues hacerlas explícitas y conseguir transformarlas es un requisito indispensable para la mejora educativa.

\section{BIBLIOGRAFÍA}

Aguilar, B. S., C. A. Mazzitelli, M. S. Chacoma y M. Aparicio, (2011). «Saberes del docente y representaciones sociales: implicancias para la enseñanza de las ciencias» en Actualidades Investigativas en Educación, vol. 11, núm. 2, 2011, pp. 1-28. Costa Rica: Universidad de Costa Rica, obtenido desde http:/ / www. redalyc.org/pdf/447/44720020007.pdf

Ertmer, P. y Newby, T. (2013). «Behaviorism, cognitivism, constructivism: comparing critical features from an instructional design perspective» en Performance Improvement Quarterly, 26(2), 43-71. 
Ferreiro, E. y Gómez Palacio, M. (comp.) (1998). Nuevas perspectivas sobre los procesos de lectura y escritura, $15^{\mathrm{a}}$ ed. México: Siglo XXI Editores.

Ferreiro Gravié, R. y Calderón M. (2000). El ABC del aprendizaje cooperativo. Trabajo en equipo para enseñar y aprender, México: Trillas.

Guirado, A, Mazzitelli, C. y Olivera, A. (2013). Representaciones sociales y práctica docente: una experiencia con profesores de física y de química. Revista de Orientación Educacional, 27(51), 87-105.

Mazzitelli, C., Aguilar, S., Guirado, A. y Olivera, A. (2009). «Representaciones sociales de los profesores sobre la docencia: contenido y estructura» en Revista Educación, Lectura y Sociedad, $6(6), 265-290$.

Moscovici, S. (1988a). Psicología social I. Influencia y cambio de actitudes. Individuos y grupos, España: Paidós. [Biblioteca Cognición y desarrollo humano; 1]

(1988b). Psicología social II. Pensamiento y vida social. Psicología social y problemas sociales, España: Paidós. [Biblioteca Cognición y desarrollo humano; 2]

Ramírez Rojas, M.I. (2016). Representaciones sociales acerca de la enseñanza de las ciencias sociales (Tesis de maestría). Universidad de Autónoma de Manizales, Colombia, obtenido desde http:/ / repositorio.autonoma.edu.co/jspui/bitstream/11182/1038/2/ RS_\%20ECS.pdf

Romero Rodríguez, E. (2004). Representaciones sociales. Atisbos y cavilaciones del devenir de cuatro décadas, México: Benemérita Universidad Autónoma de Puebla.

Secretaría de Educación Pública (2011). Programas de estudio 2011. Guía para el maestro. Educación Básica. Secundaria. Español. México. 
Strauss, A. y Corbin J. (1998). Basics of qualitative research. Techniques and procedures for developing grounded theory, 2 ed. Estados Unidos: Sage Publications.

Tyack, D. y Cuban L. (2000). En busca de la utopía. Un siglo de reformas en las escuelas públicas. México: Secretaría de Educación Pública. (Biblioteca para la actualización del maestro). 